\title{
Nonminimal couplings, gravitational waves, and torsion in Horndeski's theory
}

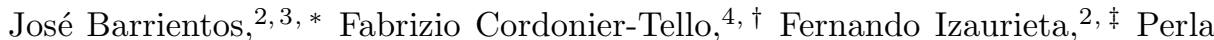

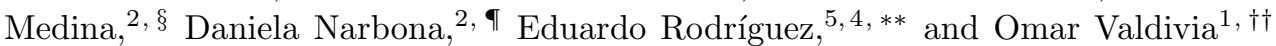 \\ ${ }^{1}$ Facultad de Ingeniería y Arquitectura, Universidad Arturo Prat, Iquique, Chile \\ ${ }^{2}$ Departamento de Física, Universidad de Concepción, Casilla 160-C, Concepción, Chile \\ ${ }^{3}$ Departamento de Enseñanza de las Ciencias Básicas, Universidad Católica del Norte, Larrondo 1281, Coquimbo, Chile \\ ${ }^{4}$ Arnold Sommerfeld Center for Theoretical Physics, Ludwig-Maximilians-Universität \\ München, Theresienstraße 37, 80333 Munich, Germany \\ ${ }^{5}$ Departamento de Fúsica, Universidad Nacional de Colombia, Bogotá, Colombia
}

(Dated: September 12, 2017)

\begin{abstract}
The Horndeski Lagrangian brings together all possible interactions between gravity and a scalar field that yield second-order field equations in four-dimensional spacetime. As originally proposed, it only addresses phenomenology without torsion, which is a non-Riemannian feature of geometry. Since torsion can potentially affect interesting phenomena such as gravitational waves and early Universe inflation, in this paper we allow torsion to exist and propagate within the Horndeski framework. To achieve this goal, we cast the Horndeski Lagrangian in Cartan's first-order formalism, and introduce wave operators designed to act covariantly on $p$-form fields that carry Lorentz indices. We find that nonminimal couplings and second-order derivatives of the scalar field in the Lagrangian are indeed generic sources of torsion. Metric perturbations couple to the background torsion and new torsional modes appear. These may be detected via gravitational waves but not through Yang-Mills gauge bosons.
\end{abstract}

PACS numbers: $04.50 .+\mathrm{h}$

Keywords: Horndeski Theory, Nonvanishing Torsion, Gravitational waves

\section{INTRODUCTION}

Recently, there has been a surge of interest in Horndeski's theory [1 [5], which is the most general fourdimensional scalar-tensor theory of gravity, without torsion, that has second-order field equations.

Torsion, however, can have dramatic effects in the very early universe [6], which is precisely one regime where scalar fields are thought to be relevant.

In the Einstein-Cartan-Sciama-Kibble (ECSK) theory [7], torsion is generated by fermions and affects only fermions. Its effects are in general very weak, since torsional terms are proportional to $\psi^{4}$ and hence only important when there is a large fermion density (see section 8.4 of Ref. 8], and Ref. 9]). Torsional effects of this magnitude will likely go undetected in any foreseeable particle physics experiment. They may be detectable in cosmological scenarios [10] and in theories that go beyond ECSK in four dimensions (see, e.g., Refs. [11 24]).

Standard model bosons, on the other hand, do not generate and are not affected by torsion 1

\footnotetext{
* josebarrientos@udec.cl

† f.cordonier@physik.uni-muenchen.de

$\ddagger$ fizaurie@udec.cl

$\S$ perlamedina@udec.cl

ฯ danielanarbona@udec.cl

** eduarodriguezsal@unal.edu.co

†† ovaldivi@unap.cl

1 This holds true when Yang-Mills bosons are described mathematically by connections on principal bundles, meaning, in particular, that the field strength reads $F_{\mu \nu}=\partial_{\mu} A_{\nu}-\partial_{\nu} A_{\mu}+$
}

Most strikingly, torsion is a nonpropagating field. In vacuum, the ECSK theory gives zero torsion, so there can be no torsional modes for gravitational waves.

The kind of nonminimal couplings between gravity and a scalar field that appear in Horndeski's theory can modify the conclusions drawn from the ECSK theory. Several authors (see, e.g., Refs. [15, 17, 18, 21]) have studied the consequences of including in the action a term that is the product of a scalar field and the Euler four-form density,

$$
L=\phi \epsilon_{a b c d} R^{a b} \wedge R^{c d},
$$

where $R^{a b}$ stands for the Lorentz curvature two-form. Here they find a twofold surprise: contrary to expectations, the term in eq. (1) produces nontrivial dynamics (in stark contrast with the uncoupled Euler density) with torsion (which normally requires fermionic fields). This term also appears naturally in several contexts (see Refs. $31-35$ ).

In this paper, we study Horndeski's theory with torsion, i.e., a theory whose relation to the original Horndeski theory is the same as that of the ECSK theory to general relativity.

Our main result can be stated as follows: Nonminimal couplings between gravity and a scalar field generically

\footnotetext{
$\frac{1}{2}\left[A_{\mu}, A_{\nu}\right]$, not $F_{\mu \nu}=\nabla_{\mu} A_{\nu}-\nabla_{\nu} A_{\mu}+\frac{1}{2}\left[A_{\mu}, A_{\nu}\right]$, which differs from the former when torsion is present. Both points of view have been studied in the literature. Some examples of the first can be found in Refs. 7, 25 27]. Some examples of the second point of view, coupling YM bosons and torsion, can be found in Refs. [28 30]. In this paper we only deal with the first approach.
} 
produce torsion. This nontrivial torsion can be thought of as an effective dark matter, which may in principle be detected via gravitational waves but not through YangMills (YM) gauge bosons.

Since torsion is a non-Riemannian feature of geometry, we find it convenient to work with Cartan's differential geometry formalism. In section [II we setup some useful definitions to deal with the Horndeski Lagrangian in the first-order formalism. We write down the Horndeski Lagrangian in its most general form and deduce its field equations from the variations with respect to the independent fields: the vierbein $e^{a}$, the spin connection $\omega^{a b}$, and the scalar field $\phi$. An interesting analysis regarding the phenomenology between some nonminimal couplings and torsion components can be found in Ref. [36]. The main difference between the section [1 of current work and Ref. [36] is that in the current article we consider the full Horndeski Lagrangian, and in Ref. [36] they explore the idea of torsion as dark energy in cosmological models.

Before plunging into an in-depth analysis of first-order perturbation theory, in section [II] we pause for a moment in order to study which torsion-aware wave operators are the most appropriate to act on our fields, which are in general differential forms that carry Lorentz indices. In this mostly mathematical section we provide a generalized version of the Weitzenböck identity that relates torsion-aware versions of the Laplace-Beltrami and the Laplace-de Rham operators, which may have an interest of its own.

In section IV we establish the first-order perturbation theory for a theory of gravity, in its first-order formalism guise, and a scalar field, and apply it to the most interesting bits of the Horndeski Lagrangian - namely, those that can lead to gravitational waves. This perturbation theory is nontrivial because, to the best of our knowledge, up until now it has been unclear how to separate the metric from the torsional degrees of freedom in the first-order perturbation of the spin connection on backgrounds with curvature and torsion. For flat backgrounds, linearized gravity in first-order formalism can be found in Ref. 37]. The separation that we achieve in eqs. (73)-(74) is novel and, while it serves as a useful step in establishing our main result-nonminimal couplings lead to nontrivial torsion that "hitches a ride" on gravitational waves-, may also find applications elsewhere.

For the sake of simplicity, in this paper we restrict ourselves to generalizing Horndeski's theory to allow for nonzero torsion, without ever going beyond second-order field equations. There exist, however, healthy theories that manage to evade the Ostrogradski instability while including higher-order derivatives (see, e.g., Refs. 38 [40]). The addition of torsion to this kind of theories remains an open problem.

\section{FIRST ORDER FORMALISM FOR HORNDESKI'S THEORY}

In this section we analyze the general behavior of the Horndeski Lagrangian without imposing the torsionless condition. The result found in Ref. 21] for the GaussBonnett term coupled to a scalar field [cf. eq. (1)] proves to be a general feature, and nonminimal couplings with an scalar field are shown to be sources of torsion.

\section{A. Preliminaries}

Let's begin with some definitions.

We shall take spacetime to be a four-dimensional smooth manifold $M$ with signature $(-+++)$. Greek indices $\mu, \nu, \ldots=0,1,2,3$ are used for tensor components in the coordinate basis, while lower-case Latin indices $a, b, \ldots=0,1,2,3$ are used for the Lorentz (orthonormal) basis. The components of the change-of-basis matrix, $e^{a}{ }_{\mu}$, help us define the one-form vierbein as $e^{a}=e^{a}{ }_{\mu} \mathrm{d} x^{\mu}$. The spacetime metric $g_{\mu \nu}$ can be written as

$$
d s^{2}=g_{\mu \nu} \mathrm{d} x^{\mu} \otimes \mathrm{d} x^{\nu}=\eta_{a b} e^{a} \otimes e^{b},
$$

whence $g_{\mu \nu}=\eta_{a b} e^{a}{ }_{\mu} e^{b}$. The space of all $p$-forms defined on $M$ is denoted as $\Omega^{p}(M)$.

It proves useful to define an operator $\Sigma_{a_{1} \cdots a_{q}}$ that maps $p$-forms into $(p-q)$-forms,

$$
\Sigma_{a_{1} \cdots a_{q}}: \Omega^{p}(M) \rightarrow \Omega^{p-q}(M),
$$

and is defined by its action on a $p$-form $\alpha$ as

$$
\Sigma^{a_{1} \cdots a_{q}} \alpha=-(-1)^{p(p-q)} *\left(e^{a_{1}} \wedge \cdots \wedge e^{a_{q}} \wedge * \alpha\right) .
$$

Here, $*$ stands for the Hodge dual, which maps $p$-forms into $(4-p)$-forms, $*: \Omega^{p}(M) \rightarrow \Omega^{4-p}(M)$.

When $q=1$ we find

$$
\Sigma^{a} \alpha=-*\left(e^{a} \wedge * \alpha\right) .
$$

This case is particularly interesting, since $\Sigma_{a}$ behaves as an exterior derivative: (i) it satisfies Leibniz's rule,

$$
\Sigma_{a}(\alpha \wedge \beta)=\Sigma_{a} \alpha \wedge \beta+(-1)^{p} \alpha \wedge \Sigma_{a} \beta,
$$

and (ii) is nilpotent,

$$
\Sigma_{a} \Sigma^{a}=0 .
$$

A key difference between $\Sigma_{a}$ and d is that, while $\mathrm{d} i n$ creases the degree of a differential form by one, $\Sigma_{a} d e$ creases it by the same amount.

In order to write the Horndeski Lagrangian in firstorder formalism (and not impose the torsionless condition from the beginning(2), we will describe the geometry

\footnotetext{
2 The torsionless Horndeski theory has already been studied in the language of differential forms; see Ref. [41].
} 
by means of the vierbein one-form $e^{a}$, the one-form spin connection $\omega^{a b}$, and the scalar field $\phi$. The spin connection and the vierbein represent independent degrees of freedom, and torsion and Lorentz curvature two-forms are given by

$$
\begin{aligned}
T^{a} & =\mathrm{D} e^{a}=\mathrm{d} e^{a}+\omega^{a}{ }_{b} \wedge e^{b}, \\
R^{a b} & =\mathrm{d} \omega^{a b}+\omega^{a}{ }_{c} \wedge \omega^{c b} .
\end{aligned}
$$

A small circle above a quantity will be used to denote the "torsionless version" of that quantity. For instance, the spin connection can always be split as

$$
\omega^{a b}=\stackrel{\circ}{\omega}^{a b}+\kappa^{a b},
$$

where $\stackrel{\circ}{\omega}^{a b}$ stands for the usual torsion-free one-form spin connection derived from the vierbein, and $\kappa^{a b}$ is the oneform contorsion. In the same way, the Lorentz curvature two-form can be expressed as

$$
R^{a b}=\stackrel{\circ}{R}^{a b}+\stackrel{\circ}{\mathrm{D}} \kappa^{a b}+\kappa_{c}^{a} \wedge \kappa^{c b},
$$

where $\stackrel{\circ}{R}^{a b}$ is the torsion-independent two-form Riemann curvature, $\stackrel{\circ}{R}^{a b}=\mathrm{d} \stackrel{\circ}{\omega}^{a b}+\stackrel{\circ}{\omega}_{c}^{a} \wedge \stackrel{\circ}{\omega}^{c b}$, and $\stackrel{\circ}{D}$ stands for the exterior covariant derivative with respect to the torsionfree connection $\stackrel{\circ}{\omega}^{a b}$.

In order to deal with the scalar field $\phi$ and its derivatives in this first-order formalism context, it proves useful to define the zero-form

$$
Z^{a}=\Sigma^{a} \mathrm{~d} \phi
$$

and the one-forms

$$
\begin{aligned}
\pi^{a} & =\mathrm{D} Z^{a}, \\
\theta^{a} & =Z^{a} \mathrm{~d} \phi .
\end{aligned}
$$

Intuitively, one can think of $Z^{a}$ as the derivative of $\phi$ in the direction specified by the $a$-index, while $\pi^{a}$ and $\theta^{a}$ represent $\partial^{2} \phi$ and $(\partial \phi)^{2}$, respectively.

\section{B. The Horndeski Lagrangian}

Using the $\Sigma_{a}$ operator and its properties, it is straightforward to work with the Horndeski Lagrangian in the first-order formalism. For instance, the "Fab Four" Lagrangians from Ref. [2] can be rewritten as the four-forms

$$
\begin{aligned}
L_{\mathrm{J}} & =\frac{1}{2} V_{\mathrm{J}}(\phi) \epsilon_{a b c d} R^{a b} \wedge e^{c} \wedge \theta^{d}, \\
L_{\mathrm{P}} & =\frac{1}{2} V_{\mathrm{P}}(\phi) \epsilon_{a b c d} R^{a b} \wedge \theta^{c} \wedge \pi^{d}, \\
L_{\mathrm{G}} & =\frac{1}{2} V_{\mathrm{G}}(\phi) \epsilon_{a b c d} R^{a b} \wedge e^{c} \wedge e^{d}, \\
L_{\mathrm{R}} & =\frac{1}{2} V_{\mathrm{R}}(\phi) \epsilon_{a b c d} R^{a b} \wedge R^{c d} .
\end{aligned}
$$

The Ringo and George cases are straightforward to translate from tensor language into differential forms, while the John and Paul cases prove more interesting. In particular, for Paul it is much more comfortable to work with $\theta^{a}$ and $\pi^{a}$ instead of the Riemann double-dual.

The same is true for the full Horndeski Lagrangian. In terms of the variables we have defined, the Horndeski Lagrangian four-form reads

$$
\begin{aligned}
L_{\mathrm{H}}(\phi, e, \omega) & =\epsilon_{a b c d}\left(2 \kappa_{1} R^{a b} \wedge e^{c} \wedge \pi^{d}+\right. \\
& +\frac{2}{3} \frac{\partial \kappa_{1}}{\partial X} \pi^{a} \wedge \pi^{b} \wedge \pi^{c} \wedge e^{d}+ \\
& +2 \kappa_{3} R^{a b} \wedge e^{c} \wedge \theta^{d}+ \\
& +2 \frac{\partial \kappa_{3}}{\partial X} \theta^{a} \wedge \pi^{b} \wedge \pi^{c} \wedge e^{d}+ \\
& +(F+2 W) R^{a b} \wedge e^{c} \wedge e^{d}+ \\
& +\frac{\partial F}{\partial X} \pi^{a} \wedge \pi^{b} \wedge e^{c} \wedge e^{d}+ \\
& +\kappa_{8} \theta^{a} \wedge \pi^{b} \wedge e^{c} \wedge e^{d}+ \\
& -\left[\frac{\partial(F+2 W)}{\partial \phi}-X \kappa_{8}\right] \pi^{a} \wedge e^{b} \wedge e^{c} \wedge e^{d}+ \\
& \left.+\kappa_{9} \frac{1}{4 !} e^{a} \wedge e^{b} \wedge e^{c} \wedge e^{d}\right)
\end{aligned}
$$

where the arbitrary functions $(i=1,3,8,9)$

$$
\begin{aligned}
\kappa_{i} & =\kappa_{i}(\phi, X), \\
F & =F(\phi, X), \\
W & =W(\phi),
\end{aligned}
$$

must satisfy the constraint

$$
\mathcal{C}(\phi, X)=\frac{\partial F}{\partial X}-2\left(\kappa_{3}+2 X \frac{\partial \kappa_{3}}{\partial X}-\frac{\partial \kappa_{1}}{\partial \phi}\right)=0
$$

with

$$
X=-\frac{1}{2} Z_{a} Z^{a}
$$

It is interesting to notice that the Hodge $*$-operator appears in the Horndeski Lagrangian exclusively through the $\Sigma^{a}$ operator. This operator allows us to cast the full Horndeski Lagrangian in an effective Lovelock-like mold [42, 43], with the Lorentz one-forms $\pi^{a}$ and $\theta^{a}$ playing a role similar to that of the vierbein, $e^{a}$.

Eq. (19) gives the full Horndeski Lagrangian in Cartan's first-order formalism. The Horndeski Theorem [1] states that, when torsion vanishes, this is the most general scalar-tensor Lagrangian that gives rise to secondorder equations for the metric. When torsion is allowed to exist, however, Horndeski's theorem is no longer valid. Indeed, it is quite easy to come up with new terms, explicitly involving torsion, that don't spoil the second-order nature of the field equations. For the sake of simplicity, in this article we will concern ourselves solely with the Horndeski Lagrangian as shown in eq. (19). The generalization of the Horndeski theorem for the case of nonvanishing torsion, i.e., the answer to the question "What is 
the most general Lagrangian that leads to second-order field equations for the metric on a spacetime with torsion?" remains an open problem and will be considered elsewhere.

\section{Field Equations}

In order to derive the field equations in the first-order paradigm, we treat $\omega^{a b}, e^{a}$, and $\phi$ as independent degrees of freedom 3

Explicitly performing the variation with respect to the spin connection yields the three-form equation $\mathcal{E}_{a b}=0$, where

$$
\begin{aligned}
\mathcal{E}_{a b} & =-\epsilon_{a b c d} T^{c} \wedge\left[\kappa_{1} \pi^{d}+\kappa_{3} \theta^{d}+(F+2 W) e^{d}\right]+ \\
& +\epsilon_{a b c d} e^{c} \wedge\left[\mathrm{d} \kappa_{1} \wedge \pi^{d}+\kappa_{1} R_{e}^{d} Z^{e}+\mathrm{d} \kappa_{3} \wedge \theta^{d}+\right. \\
& \left.-\kappa_{3} \mathrm{~d} \phi \wedge \pi^{d}+\frac{1}{2} \mathrm{~d}(F+2 W) \wedge e^{d}\right]+ \\
& -\frac{1}{2}\left(Z_{a} \epsilon_{b c d e}-Z_{b} \epsilon_{a c d e}\right)\left[\kappa_{1} R^{c d}+\right. \\
& +\pi^{c} \wedge\left(\frac{\partial \kappa_{1}}{\partial X} \pi^{d}+2 \frac{\partial \kappa_{3}}{\partial X} \theta^{d}+\frac{\partial F}{\partial X} e^{d}\right)+ \\
& \left.+\frac{1}{2}\left(\kappa_{8} \theta^{c}-\left[\frac{\partial}{\partial \phi}(F+2 W)-X \kappa_{8}\right] e^{c}\right) \wedge e^{d}\right] \wedge e^{e}
\end{aligned}
$$

The field equations obtained from variation with respect to the vierbein and the scalar field, on the other hand, read

$$
\begin{aligned}
\mathcal{E}_{a} & =E_{a}+\Sigma^{b}\left(\mathcal{S}_{b}+\mathcal{T}_{b}+\mathcal{U}_{b}\right) Z_{a}=0 \\
\mathcal{E} & =E+\mathcal{Z}-\mathrm{d} \Sigma^{b}\left(\mathcal{S}_{b}+\mathcal{T}_{b}+\mathcal{U}_{b}\right)=0
\end{aligned}
$$

where

$$
\begin{aligned}
E_{d} & =\epsilon_{a b c d}\left(2 \kappa_{1} R^{a b} \wedge \pi^{c}+\frac{2}{3} \frac{\partial \kappa_{1}}{\partial X} \pi^{a} \wedge \pi^{b} \wedge \pi^{c}+\right. \\
& +2 \kappa_{3} R^{a b} \wedge \theta^{c}+2 \frac{\partial \kappa_{3}}{\partial X} \theta^{a} \wedge \pi^{b} \wedge \pi^{c}+ \\
& +2(F+2 W) R^{a b} \wedge e^{c}+2 \frac{\partial F}{\partial X} \pi^{a} \wedge \pi^{b} \wedge e^{c}+ \\
& +2 \kappa_{8} \theta^{a} \wedge \pi^{b} \wedge e^{c}+\frac{1}{3 !} \kappa_{9} e^{a} \wedge e^{b} \wedge e^{c}+ \\
& \left.-3\left[\frac{\partial}{\partial \phi}(F+2 W)-X \kappa_{8}\right] \pi^{a} \wedge e^{b} \wedge e^{c}\right),
\end{aligned}
$$

\footnotetext{
${ }^{3}$ Note that $Z^{a}$ depends on $e^{a}$ and the derivatives of $\phi$ through the $\Sigma^{a}$ operator. This dependence must be taken into account when performing the variations with respect to $e^{a}$ and $\phi$.
}

$$
\begin{aligned}
& E=\epsilon_{a b c d}\left[2\left(\frac{\partial \kappa_{1}}{\partial \phi}-\kappa_{3}\right) R^{a b} \wedge e^{c} \wedge \pi^{d}+\right. \\
& +2\left(\frac{1}{3} \frac{\partial^{2} \kappa_{1}}{\partial \phi \partial X}-\frac{\partial \kappa_{3}}{\partial X}\right) \pi^{a} \wedge \pi^{b} \wedge \pi^{c} \wedge e^{d}+ \\
& +2 \frac{\partial \kappa_{3}}{\partial \phi} R^{a b} \wedge e^{c} \wedge \theta^{d}+ \\
& +2 \frac{\partial^{2} \kappa_{3}}{\partial \phi \partial X} \theta^{a} \wedge \pi^{b} \wedge \pi^{c} \wedge e^{d}+ \\
& +\left(\frac{\partial F}{\partial \phi}+2 \frac{\partial W}{\partial \phi}\right) R^{a b} \wedge e^{c} \wedge e^{d}+ \\
& +\left(\frac{\partial^{2} F}{\partial \phi \partial X}-\kappa_{8}\right) \pi^{a} \wedge \pi^{b} \wedge e^{c} \wedge e^{d}+ \\
& +\frac{\partial \kappa_{8}}{\partial \phi} \theta^{a} \wedge \pi^{b} \wedge e^{c} \wedge e^{d}+ \\
& -\left[\frac{\partial^{2}(F+2 W)}{\partial \phi^{2}}-X \frac{\partial \kappa_{8}}{\partial \phi}\right] \pi^{a} \wedge e^{b} \wedge e^{c} \wedge e^{d}+ \\
& \left.+\frac{1}{4 !} \frac{\partial \kappa_{9}}{\partial \phi} e^{a} \wedge e^{b} \wedge e^{c} \wedge e^{d}\right] \\
& \mathcal{Z}=\left[2 \mathrm{~d} \kappa_{3} \wedge R^{a b}+2 \mathrm{~d} \frac{\partial \kappa_{3}}{\partial X} \wedge \pi^{a} \wedge \pi^{b}+\right. \\
& +\mathrm{d} \kappa_{8} \wedge \pi^{a} \wedge e^{b}+ \\
& \left.+\mathrm{D} \pi^{a} \wedge\left(4 \frac{\partial \kappa_{3}}{\partial X} \pi^{b}+\kappa_{8} e^{b}\right)\right] \wedge e^{c} Z^{d}+ \\
& +2 \epsilon_{\text {abcd }}\left[\kappa_{3} R^{a b}+\frac{\partial \kappa_{3}}{\partial X} \pi^{a} \wedge \pi^{b}+\right. \\
& \left.+\kappa_{8} \pi^{a} \wedge e^{b}\right] \wedge T^{c} Z^{d},
\end{aligned}
$$

and the common 4 -form variables $\mathcal{S}_{a}, \mathcal{T}_{a}$ and $\mathcal{U}_{a}$ are given by

$$
\begin{aligned}
\mathcal{S}_{d} & =2 \epsilon_{a b c d}\left[\mathrm{D} \pi^{a} \wedge e^{b} \wedge\left(2 \frac{\partial \kappa_{1}}{\partial X} \pi^{c}+2 \frac{\partial \kappa_{3}}{\partial X} \theta^{c}+\frac{\partial F}{\partial X} e^{c}\right)+\right. \\
+ & \pi^{a} \wedge e^{b} \wedge \mathrm{d} X \wedge\left(\frac{\partial^{2} \kappa_{1}}{\partial X^{2}} \pi^{c}+2 \frac{\partial^{2} \kappa_{3}}{\partial X^{2}} \theta^{c}+\frac{\partial^{2} F}{\partial X^{2}} e^{c}\right)+ \\
+ & \left.\frac{1}{2} e^{a} \wedge e^{b} \wedge \mathrm{d} X \wedge\left(\theta^{c} \frac{\partial \kappa_{8}}{\partial X}-e^{c} \frac{\partial}{\partial X}\left\{\frac{\partial F}{\partial \phi}-X \kappa_{8}\right\}\right)\right] \\
\mathcal{T}_{d} & =2 \epsilon_{a b c d}\left[\kappa_{1} R^{a b}+\frac{\partial \kappa_{1}}{\partial X} \pi^{a} \wedge \pi^{b}+2 \frac{\partial \kappa_{3}}{\partial X} \pi^{a} \wedge \theta^{b}+\right. \\
& +2 \frac{\partial F}{\partial X} \pi^{a} \wedge e^{b}+\frac{1}{2} \kappa_{8} e^{a} \wedge \theta^{b}+ \\
& -\frac{3}{2}\left(\frac{\partial}{\partial \phi}(F+2 W)-X^{b}\right) e^{a} \wedge e^{b} \wedge T^{c} \\
& \left.+\pi^{a} \wedge e^{b} \wedge e^{c} \wedge M_{e}^{d}+e^{a} \wedge e^{b} \wedge e^{c} \wedge K_{e}^{d}\right] \\
\mathcal{U}_{e} & =\epsilon_{a b c d}\left[-R^{a b} \wedge e^{c} \wedge\left(C_{e}^{d}+2 \frac{\partial \kappa_{1}}{\partial X} \delta_{e f}^{g d} Z_{g} \pi^{f}\right)+\right. \\
& -\pi^{a} \wedge \pi^{b} \wedge e^{c} \wedge\left(\bar{C}_{e}^{d}+\frac{2}{3} \frac{\partial^{2} \kappa_{1}}{\partial X^{2}} \pi^{d} Z_{e}\right)+ \\
&
\end{aligned}
$$


In eq. (33), $C_{b}^{a}, \bar{C}_{b}^{a}, K_{b}^{a}$, and $M_{b}^{a}$ are one-forms defined as

$$
\begin{aligned}
C_{b}^{a}{ }_{b} & =2 \mathrm{~d} \phi\left[\frac{\partial \kappa_{3}}{\partial X} Z^{a} Z_{b}-\left(\kappa_{3}-\frac{\partial \kappa_{1}}{\partial \phi}\right) \delta_{b}^{a}\right]+ \\
& +e^{a} Z_{b} \frac{\partial F}{\partial X} \\
\bar{C}_{b}^{a} & =2 \mathrm{~d} \phi\left[\frac{\partial^{2} \kappa_{3}}{\partial X^{2}} Z^{a} Z_{b}-\left(3 \frac{\partial \kappa_{3}}{\partial X}-\frac{\partial^{2} \kappa_{1}}{\partial \phi \partial X}\right) \delta_{b}^{a}\right]+ \\
& +e^{a} Z_{b} \frac{\partial^{2} F}{\partial X^{2}} \\
K_{b}^{a} & =\left[\frac{\partial^{2}}{\partial \phi^{2}}(F+2 W)-X \frac{\partial \kappa_{8}}{\partial \phi}\right] \mathrm{d} \phi \delta_{b}^{a}+ \\
& -\frac{1}{4 !} e^{a} Z_{b} \frac{\partial \kappa_{9}}{\partial X}, \\
M_{b}^{a} & =\left(2\left[\kappa_{8}-\frac{\partial^{2} F}{\partial \phi \partial X}\right] \delta_{b}^{a}-\frac{\partial \kappa_{8}}{\partial X} Z^{a} Z_{b}\right) \mathrm{d} \phi+ \\
& +e^{a} Z_{b} \frac{\partial}{\partial X}\left[\frac{\partial F}{\partial \phi}-X \kappa_{8}\right]
\end{aligned}
$$

The one-forms $C^{a}{ }_{b}$ and $\bar{C}^{a}{ }_{b}$ satisfy the properties

$$
\begin{aligned}
\Sigma^{b} C^{a}{ }_{b} & =Z^{a} \mathcal{C}, \\
\Sigma^{b} \bar{C}^{a}{ }_{b} & =Z^{a} \frac{\partial \mathcal{C}}{\partial X},
\end{aligned}
$$

where $\mathcal{C}(\phi, X)=0$ is the Horndeski constraint (23).

Here it is important to observe that in the terms $\mathcal{Z}$ and $\mathcal{T}_{a}$ torsion appears explicitly as a result of nonminimal couplings. Torsional degrees of freedom are also contained inside the Lorentz curvature through the contorsion one-form, as shown in eq. (11).

As the quickest glance at eqs. (25)-(37) will show, the full Horndeski theory is extremely complicated. Actually, it may be more accurate to think of it as a family of theories, each one defined by a choice of the arbitrary functions $\kappa_{i}, F$, and $W$. There are, however, several general observations to be made.

First, using the properties of the $\Sigma^{a}$ operator it is straightforward to derive the field equations obtained from the independent variations of $e^{a}$ and $\omega^{a b}$, without imposing the torsionless condition. Trying to achieve the same feat in the standard Palatini tensor formalism would have been impractical, to say the least.

Second, the field equations directly show that torsion arises, in general, from every nonminimal coupling with the scalar field, and from the terms depending on $\pi^{a}=\mathrm{D} \Sigma^{a} \mathrm{~d} \phi$. For instance, see the term $\mathcal{T}_{a}$ in eqs. (26) and (27), and the first term of eq. (25).

In order to recover the standard torsionless dynamics, one cannot simply impose $T^{a}=0$ on the equations of motion. This happens because, in the general setting, the dynamics of $\phi$ and the torsion become fully intertwined, generically leading to $T^{a} \sim \partial \phi$. Therefore, imposing $T^{a}=0$ in these cases will lead to $\phi=$ const., freezing the dynamics of the scalar field. The important point here is that the standard torsionless case corresponds to a constraint on the more general Cartan geometry framework. This problem seems to have been known for a long time; see, e.g., Ref. [44], or section 1.7.1 of Ref. [45]. The solution for it can be be written in a very practical way in terms of the $\Sigma^{a}$ operator. First, we have to include the torsionless condition via a two-form Lagrange multiplier with a Lorentz index, $\Lambda_{a}$,

$$
L_{\mathrm{H}} \rightarrow \bar{L}_{\mathrm{H}}=L_{\mathrm{H}}+\Lambda_{a} \wedge T^{a},
$$

whence we get the new equations of motion

$$
\begin{aligned}
\overline{\mathcal{E}}_{a} & =\mathcal{E}_{a}-\mathrm{D} \Lambda_{a}=0, \\
\overline{\mathcal{E}} & =\mathcal{E}=0, \\
\overline{\mathcal{E}}^{a b} & =\mathcal{E}^{a b}-\frac{1}{2}\left(\Lambda^{a} \wedge e^{b}-\Lambda^{b} \wedge e^{a}\right)=0, \\
T^{a} & =0 .
\end{aligned}
$$

Using the $\Sigma^{a}$ operator, it is possible to solve $\overline{\mathcal{E}}^{a b}=0$ for $\Lambda_{a}$. We find

$$
\Lambda^{a}=2 \Sigma_{b} \mathcal{E}^{a b}+\frac{1}{2} e^{a} \wedge \Sigma_{b c} \mathcal{E}^{b c} .
$$

Therefore, the standard field equations for the torsionless Horndeski theory are recovered in this setting as

$$
\begin{aligned}
\mathcal{E}^{a}-2 \mathrm{D} \Sigma_{b} \mathcal{E}^{a b}+\left.\frac{1}{2} e^{a} \wedge \mathrm{d} \Sigma_{b c} \mathcal{E}^{b c}\right|_{T^{a}=0} & =0 \\
\left.\mathcal{E}\right|_{T^{a}=0} & =0 .
\end{aligned}
$$

This behavior is in stark contrast with the standard Einstein-Cartan case with minimally coupled fields. In this case, $T^{a}=0$ is an equation of motion in vacuum, and therefore it is unnecessary to use a Lagrange multiplier. In fact, in this case only fermionic fields can be a source of non-propagating torsion (see, e.g., section 8.4 of Ref. [8]).

Known results for the torsionless Horndeski theory encompass from cosmological models to black hole solutions. In the torsional Horndeski setting here presented, such solutions might persist [15, 17] if one uplifts them in a consistent manner. This means, in particular, that the Riemann curvature and the scalar field should be the same as in the torsionless theory. Torsion, however, must be present if the scalar field is nontrivial, and this will generically imply that the Lorentz curvature shall differ from the Riemann curvature [46, 47]. Because of the complexity of Horndeski's theory, it seems unlikely that one can make a general statement as to whether this uplifting can always be done, or which conditions must be fulfilled for it to succeed.

\section{WAVE OPERATORS, TORSION, AND THE WEITZENBÖCK IDENTITY}

Our goal in this section is to define a wave operator that can act on differential forms that carry Lorentz indices, such as the vierbein, $e^{a}$. We need this operator 
because our treatment of gravitational waves relies on perturbations of the vierbein and the spin connection, $\omega^{a b}$, which are the natural independent degrees of freedom for a spacetime with torsion.

TABLE I. Many different derivatives are defined in this section. This table collects all definitions and some of their most important properties.

\begin{tabular}{|c|c|c|c|}
\hline Symbol & Definition & $\begin{array}{c}\text { Change in } \\
\text { form degree }\end{array}$ & $\begin{array}{c}\text { Key } \\
\text { property }\end{array}$ \\
\hline $\mathrm{d}$ & $\overline{\mathrm{d} x^{\mu} \partial_{\mu}}$ & +1 & $d^{2}=0$ \\
\hline D & $d+\omega$ & +1 & \\
\hline$\stackrel{D}{D}$ & $d+\stackrel{\omega}{\omega}$ & +1 & \\
\hline $\mathrm{d}^{\dagger}$ & $* \mathrm{~d} *$ & -1 & \\
\hline $\mathrm{D}^{\dagger}$ & $* \mathrm{D} *$ & -1 & \\
\hline$\stackrel{\circ}{D}^{\dagger}$ & $* \stackrel{\circ}{D} *$ & -1 & \\
\hline$\Sigma^{a}$ & $-*\left(e^{a} \wedge *\right.$ & -1 & $\Sigma^{a} \Sigma_{a}=0$ \\
\hline $\mathrm{D}^{\ddagger}$ & $-\Sigma^{a} \mathrm{D} \Sigma_{a}$ & -1 & \\
\hline$\stackrel{\circ}{ }^{\ddagger}$ & $-\Sigma^{a} \stackrel{\circ}{\mathrm{D}} \Sigma_{a}$ & -1 & 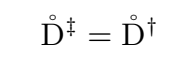 \\
\hline $\mathcal{D}_{a}$ & $\Sigma_{a} \mathrm{D}+\mathrm{D} \Sigma_{a}$ & 0 & \\
\hline$\stackrel{\circ}{\mathcal{D}}_{a}$ & $\Sigma_{a} \mathrm{D}+\stackrel{\circ}{D} \Sigma_{a}$ & 0 & $\stackrel{\circ}{\mathcal{D}}_{a}=e_{a}{ }^{\mu} \stackrel{\circ}{\nabla}_{\mu}$ \\
\hline$\square_{\mathrm{dR}}$ & $\mathrm{d}^{\dagger} \mathrm{d}+\mathrm{dd}^{\dagger}$ & 0 & \\
\hline$\square_{\mathrm{B}}$ & $-\stackrel{\circ}{\nabla}^{\mu} \stackrel{\circ}{\nabla}_{\mu}$ & 0 & \\
\hline $\mathbf{\square}_{\mathrm{dR}}$ & $\mathrm{DD}^{\ddagger}+\mathrm{D}^{\ddagger} \mathrm{D}$ & 0 & \\
\hline $\mathbf{a}_{\mathrm{B}}$ & $-\mathcal{D}^{a} \mathcal{D}_{a}$ & 0 & \\
\hline
\end{tabular}

Let $\Phi$ be a scalar (i.e., without Lorentz indices) $p$-form,

$$
\Phi=\frac{1}{p !} \Phi_{\mu_{1} \cdots \mu_{p}} \mathrm{~d} x^{\mu_{1}} \wedge \cdots \wedge \mathrm{d} x^{\mu_{p}} .
$$

There are at least two wave operators that can conceivably act on $\Phi$. The Laplace-de Rham operator,

$$
\square_{\mathrm{dR}}=\mathrm{d}^{\dagger} \mathrm{d}+\mathrm{dd}^{\dagger}
$$

is defined as the anticommutator of the exterior derivative and the exterior coderivative, $\mathrm{d}^{\dagger}=* \mathrm{~d} *$ [for dimensions other than four or signatures other than $(-+++)$, the definition of $\mathrm{d}^{\dagger}$ must be modified with a judiciously chosen sign]. This operator satisfies the Weitzenböck identity

$$
\square_{\mathrm{dR}} \Phi=\square_{\mathrm{B}} \Phi+\Sigma_{a}\left(\stackrel{\circ}{R}_{b}^{a} \wedge \Sigma^{b} \Phi\right),
$$

where $\square_{\mathrm{B}}=-\stackrel{\circ}{\nabla}^{\mu} \stackrel{\circ}{\nabla}_{\mu}$ is the usual Laplace-Beltrami operator built from the torsion-free covariant derivative $\stackrel{\circ}{\nabla}_{\mu}$. While unconventional, writing the Weitzenböck identity as in eq. (50) proves to be useful for our purposes and is equivalent to more common approaches. In words, the Weitzenböck identity states that the difference between the two wave operators acting on $\Phi$ is related to the curvature of the manifold and does not involve derivatives of $\Phi$.

By definition, the Laplace-Beltrami operator carries no information about torsion. Since it is the Riemann curvature two-form, $\stackrel{\circ}{R}^{a b}$, that appears in the second term on the right-hand side of eq. (50), this means that eq. (50) has no information at all about torsion, even when torsion is present in spacetime. This is consistent with the fact that the Laplace-de Rham operator is defined without any reference to torsion.

An example of the usefulness of this construction is provided by classical electromagnetism on a curved spacetime. Let $A$ be the electromagnetic potential oneform and $F=\mathrm{d} A$ its associated field strength two-form. Maxwell equations in vacuum can be written as

$$
\mathrm{d}^{\dagger} F=\mathrm{d}^{\dagger} \mathrm{d} A=0 .
$$

Choosing the Lorenz gauge, $\mathrm{d}^{\dagger} A=0$, we can use eq. (50) to find

$$
\square_{\mathrm{B}} A+\Sigma_{a} \stackrel{\circ}{R}_{b}^{a} \Sigma^{b} A=0,
$$

or, in standard tensor language,

$$
-\stackrel{\circ}{\nabla}^{\lambda} \stackrel{\circ}{\nabla}_{\lambda} A_{\mu}+\stackrel{\circ}{R}_{\mu \nu} A^{\nu}=0,
$$

where $\stackrel{\circ}{R}_{\mu \nu}$ is the standard torsionless Ricci tensor. It is interesting to notice that this result holds even when the background geometry has nonvanishing torsion. The electromagnetic field only interacts with the torsionless sector of the geometry. The same happens with all YM gauge bosons: they only can interact with the torsionless sector of the geometry

Extending the de Rham definition of the wave operator for the case of a $p$-form with $m$ free Lorentz indices, such as

$$
\Psi^{a_{1} \cdots a_{m}}=\frac{1}{p !} \Psi_{\mu_{1} \cdots \mu_{p}}^{a_{1} \cdots a_{m}} \mathrm{~d} x^{\mu_{1}} \wedge \cdots \wedge \mathrm{d} x^{\mu_{p}},
$$

is nontrivial when the geometry has nonvanishing torsion. As a first step, one may be inclined to define the de Rham Lorentz-covariant coderivative as $\mathrm{D}^{\dagger}=* \mathrm{D} *$, in perfect analogy with $\mathrm{d}^{\dagger}=* \mathrm{~d} *$. We find, however, that a more useful definition is

$$
\mathrm{D}^{\ddagger}=-\Sigma^{a} \mathrm{D} \Sigma_{a} .
$$

This is equivalent to the first definition when torsion is zero,

$$
* \stackrel{\circ}{ } *=-\Sigma^{a} \stackrel{\circ}{\mathrm{D}} \Sigma_{a},
$$

but not in general. What makes definition (55) useful is that the wave operator built from it satisfies a generalized version of the Weitzenböck identity (50).

Let us define the generalized Laplace-de Rham operator as

$$
\mathbf{\square}_{\mathrm{dR}}=\mathrm{DD}^{\ddagger}+\mathrm{D}^{\ddagger} \mathrm{D} \text {. }
$$

\footnotetext{
${ }^{4}$ See footnote 1 on page 1
} 
It is possible to prove that $\mathbf{}_{\mathrm{dR}}$ satisfies the following generalized Weitzenböck identity:

$$
\begin{aligned}
\mathbf{\square}_{\mathrm{dR}} \Phi^{a_{1} \cdots a_{m}} & =\mathbf{\square}_{\mathrm{B}} \Phi^{a_{1} \cdots a_{m}}+\Sigma_{c} \mathrm{D}^{2} \Sigma^{c} \Phi^{a_{1} \cdots a_{m}} \\
& =\mathbf{\square}_{\mathrm{B}} \Phi^{a_{1} \cdots a_{m}}+\Sigma_{c}\left(R^{c}{ }_{b} \Sigma^{b} \Phi^{a_{1} \cdots a_{m}}+\right. \\
& +R^{a_{1}}{ }_{b} \Sigma^{c} \Phi^{b a_{2} \cdots a_{m}}+\cdots+ \\
& \left.+R^{a_{m}}{ }_{b} \Sigma^{c} \Phi^{a_{1} \cdots a_{m-1} b}\right) .
\end{aligned}
$$

In eq. (58) we have introduced the generalized LaplaceBeltrami operator

$$
\mathbf{\square}_{\mathrm{B}}=-\mathcal{D}^{a} \mathcal{D}_{a}
$$

where

$$
\mathcal{D}_{a}=\Sigma_{a} \mathrm{D}+\mathrm{D} \Sigma_{a}
$$

In the torsionless case, the operator $\mathcal{\mathcal { D }}_{a}=\Sigma_{a} \stackrel{\circ}{\mathrm{D}}+\stackrel{\circ}{\mathrm{D}} \Sigma_{a}$ can be shown to satisfy $\stackrel{\circ}{\mathcal{D}}_{a}=e_{a}{ }^{\mu} \stackrel{\circ}{\nabla}_{\mu}$, meaning that it matches the usual torsionless covariant derivative $\stackrel{\circ}{\nabla}^{\circ}=$ $\partial+\stackrel{\circ}{\Gamma}$, and the standard Weitzenböck identity (50) is recovered.

Some useful properties satisfied by $\mathcal{D}_{a}$ are

$$
\begin{aligned}
\mathcal{D}_{a}(\alpha \wedge \beta) & =\mathcal{D}_{a} \alpha \wedge \beta+\alpha \wedge \mathcal{D}_{a} \beta \\
{\left[\Sigma_{a}, \mathcal{D}_{b}\right] } & =-\left(\Sigma_{a b} T^{c}\right) \Sigma_{c} \\
{\left[\mathcal{D}_{a}, \mathcal{D}_{b}\right] } & =\mathrm{D}^{2} \Sigma_{a b}+\Sigma_{a b} \mathrm{D}^{2}+\Sigma_{a} \mathrm{D}^{2} \Sigma_{b}-\Sigma_{b} \mathrm{D}^{2} \Sigma_{a}+ \\
& -\left(\mathrm{D} \Sigma_{a b} T^{c}\right) \wedge \Sigma_{c}-\left(\Sigma_{a b} T^{c}\right) \mathcal{D}_{c}
\end{aligned}
$$

where $\alpha$ is a $p$-form and $\beta$ is a $q$-form. In particular, eq. (61) implies that $\mathcal{D}_{a}$ obeys Leibniz's rule without any correcting signs.

From the above discussion, it seems clear that in order to have waves interacting with torsion, it is necessary for the field to have free Lorentz indices. This is precisely the case of gravitational waves in the Horndeski case, as we shall see in the next section.

\section{GRAVITATIONAL WAVES AND TORSIONAL MODES}

\section{A. Linear perturbations for a theory of gravity in the first-order formalism}

Let us consider a background geometry described by $\bar{e}^{a}, \bar{\omega}^{a b}$ and $\bar{\phi}$. Linear perturbation 5 around this back-

\footnotetext{
${ }^{5}$ It is very important to remember that in order to study cases of astrophysical interest, it is necessary to go at least to secondorder in the perturbations of curvature. In the current article we are not interested in modelling a particular phenomena, but just studying how gravitational waves could interact with torsion at first-order. Detailed calculations to second-order for particular astrophysical situations will be presented elsewhere.
}

ground are described by

$$
\begin{aligned}
\bar{e}^{a} \rightarrow e^{a} & =\bar{e}^{a}+\frac{1}{2} h^{a}, \\
\bar{\omega}^{a b} \rightarrow \omega^{a b} & =\bar{\omega}^{a b}+u^{a b}, \\
\bar{\phi} \rightarrow \phi & =\bar{\phi}+\varphi,
\end{aligned}
$$

where we have introduced the one-forms $h^{a}=h^{a}{ }_{b} \bar{e}^{b}$ and $u^{a b}=u^{a b}{ }_{c} \bar{e}^{c}$, and the zero-form $\varphi$.

The linear perturbation of the metric reads

$$
\begin{aligned}
g & =\eta_{a b} e^{a} \otimes e^{b} \\
& =\eta_{a b}\left(\bar{e}^{a}+\frac{1}{2} h^{a}{ }_{c} \bar{e}^{c}\right) \otimes\left(\bar{e}^{b}+\frac{1}{2} h_{{ }_{d}}^{b} \bar{e}^{d}\right), \\
& =\eta_{a b} \bar{e}^{a} \otimes \bar{e}^{b}+\frac{1}{2}\left(h_{a b}+h_{b a}\right) \bar{e}^{a} \otimes \bar{e}^{b}, \\
& =\left(\bar{g}_{\mu \nu}+h_{\mu \nu}^{+}\right) \mathrm{d} x^{\mu} \otimes \mathrm{d} x^{\nu},
\end{aligned}
$$

where

$$
h_{a b}^{ \pm}=\frac{1}{2}\left(h_{a b} \pm h_{b a}\right)
$$

are the symmetric and antisymmetric parts of $h_{a b}$. The standard theory of gravitational waves is formulated just in terms of $h_{\mu \nu}^{+}$, because it is possible to show that the antisymmetric part, $h_{a b}^{-}$, amounts to nothing more than an infinitesimal local Lorentz transformation. Since the Horndeski Lagrangian (19) is locally Lorentz invariant, it is possible to gauge away that piece and to keep only the symmetric part. Therefore, from now on we will just assume that $h_{a b}$ is symmetric, i.e., $h_{b a}=h_{a b}$.

In standard general relativity, the perturbation in the geometry is described in terms of $h_{\mu \nu}$ alone, since the perturbation in the connection depends on the $h_{\mu \nu}$ through the torsionless condition. When considering nonvanishing torsion, the vierbein and the spin connection correspond to independent degrees of freedom. Therefore, the perturbation one-forms $h^{a}$ and $u^{a b}$ must be independent, too. Here we show that it is always possible to split the linear perturbation one-form $u^{a b}$ in two pieces, one carrying all the dependency on $h^{a}$ and one completely independent from it (and associated, of course, to linear perturbations in the torsion).

Let us begin by writing down the two-form torsion as $T^{a}=\mathrm{D} e^{a}$. Its linear perturbation under eqs. (64)- (66) is given by

$$
\begin{aligned}
\bar{T}^{a} \rightarrow T^{a} & =\bar{T}^{a}+\frac{1}{2} \overline{\mathrm{D}} h^{a}+u_{b}^{a} \wedge \bar{e}^{b}, \\
& =\bar{T}^{a}+\frac{1}{2} \overline{\mathrm{D}} h^{a}+\frac{1}{2} \bar{\kappa}_{b}^{a} \wedge h^{b}+u_{b}^{a} \wedge \bar{e}^{b},
\end{aligned}
$$

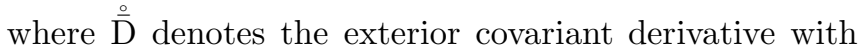
respect to the torsionless piece of the background spin connection, $\stackrel{\circ}{\omega}^{a b}$.

On the other hand, torsion may be also written in terms of the contorsion one-form, $\kappa^{a b}$, as $T^{a}=\kappa_{b}^{a} \wedge e^{b}$. 
Its linear perturbation reads

$$
\bar{T}^{a} \rightarrow T^{a}=\bar{T}^{a}+\frac{1}{2} \kappa_{b}^{a} \wedge h^{b}+q_{b}^{a} \wedge \bar{e}^{b},
$$

where $q^{a b}$ stands for the linear perturbation in the contorsion, i.e., $\bar{\kappa}^{a b} \rightarrow \kappa^{a b}=\bar{\kappa}^{a b}+q^{a b}$.

Equations (69) and (70) may seem contradictory at first sight, since one of them includes derivatives of $h^{a}$ and the other doesn't. There is no contradiction, though; to see this, one need only notice that $u^{a b}$ must be of the form

$$
u^{a b}=\stackrel{\circ}{a b}^{a b}+q^{a b}
$$

where

$$
\frac{1}{2} \stackrel{\circ}{\mathrm{D}} h^{a}+\stackrel{\circ}{u}_{b}{ }_{b} \wedge \bar{e}^{b}=0 .
$$

Using eqs. (71) and (72) in eq. (69), the apparent contradiction is resolved.

In order to avoid an algebraic nightmare where both $\stackrel{\circ}{\bar{D}}$ and $\overline{\mathrm{D}}$ derivatives get mixed together, it is convenient to define the new perturbation variables

$$
\begin{aligned}
& \mathcal{U}_{a b}=\dot{u}_{a b}-\frac{1}{2}\left[\bar{\Sigma}_{a}\left(\bar{\kappa}_{b c} \wedge h^{c}\right)-\bar{\Sigma}_{b}\left(\bar{\kappa}_{a c} \wedge h^{c}\right)\right] \\
& \mathcal{V}_{a b}=q_{a b}+\frac{1}{2}\left[\bar{\Sigma}_{a}\left(\bar{\kappa}_{b c} \wedge h^{c}\right)-\bar{\Sigma}_{b}\left(\bar{\kappa}_{a c} \wedge h^{c}\right)\right] .
\end{aligned}
$$

These clearly satisfy

$$
u^{a b}=\stackrel{\circ}{a b}^{a b} q^{a b}=\mathcal{U}^{a b}+\mathcal{V}^{a b} .
$$

Using the fact that torsion and contorsion are related by

$$
\kappa_{a b}=\frac{1}{2}\left(\Sigma_{a} T_{b}-\Sigma_{b} T_{a}+e^{c} \Sigma_{a b} T_{c}\right),
$$

one can show that eq. (72) becomes

$$
\frac{1}{2} \overline{\mathrm{D}} h^{a}+\mathcal{U}_{b}^{a} \wedge \bar{e}^{b}+\frac{1}{2} \bar{\Sigma}^{a}\left(h_{b} \wedge \bar{T}^{b}\right)=0,
$$

and that eq. (70) becomes the torsion linear perturbation equation

$$
\bar{T}^{a} \rightarrow T^{a}=\bar{T}^{a}+\mathcal{V}_{b}^{a} \wedge \bar{e}^{b}-\frac{1}{2} \bar{\Sigma}^{a}\left(h_{b} \wedge \bar{T}^{b}\right) .
$$

From eq. (77), and after some algebra, it is possible to get a closed expression for $\mathcal{U}^{a b}$,

$$
\mathcal{U}^{a b}=-\frac{1}{2}\left(\bar{\Sigma}^{a} \overline{\mathrm{D}} h^{b}-\bar{\Sigma}^{b} \overline{\mathrm{D}} h^{a}\right)
$$

The linear perturbation of the Lorentz curvature reads simply

$$
\bar{R}^{a b} \rightarrow R^{a b}=\bar{R}^{a b}+\overline{\mathrm{D}}\left(\mathcal{U}^{a b}+\mathcal{V}^{a b}\right) .
$$

We have thus been able to split the Lorentz connection perturbation, $u^{a b}$, in two parts, $\mathcal{U}^{a b}$ and $\mathcal{V}^{a b}$, such that
$\mathcal{U}^{a b}$ is completely determined by the vierbein perturbation [via eq. (79)], and the linear perturbation on the torsion depends only on $\mathcal{V}^{a b}$ [cf. eq. (78)]. The Lorentz curvature perturbation, on the other hand, depends on both parts of the Lorentz connection perturbation, as shown in eq. (80).

Finally, one can show that the scalar field "curvature" $Z^{a}=\Sigma^{a} \mathrm{~d} \phi$ becomes

$$
\bar{Z}^{a} \rightarrow Z^{a}=\bar{Z}^{a}+\bar{\Sigma}^{a} \mathrm{~d} \varphi-\frac{1}{2} h_{b}^{a} \bar{Z}^{b} .
$$

\section{B. Gravitational Waves and Torsion in Horndeski's Theory}

As we have seen in section II nonminimal couplings and second-order derivatives terms in the Horndeski lagrangian are sources of torsion. In this general case, torsion propagates through the "contorsional mode" $\mathcal{V}^{a b}$ and the background torsion $\bar{T}^{a}$ interacts with the metric modes $h^{a}$. However, our intuition may lead us to believe that the Einstein-Hilbert $(\mathrm{EH})$ term can give rise only to the wave equation and interactions of $h^{a}$ with the background curvature, as in the standard torsionless case. That is not the case. As we shall see, even the EH term gives rise to both, metrical modes interacting with the background torsion and propagating torsional modes.

In order to see this, let us consider a Lagrangian in the Horndeski family of the form

$$
\mathcal{L}^{(4)}(e, \omega, \phi)=\mathcal{L}_{\mathrm{EH}}^{(4)}+(\text { other terms }),
$$

where these "other terms" are the ones giving rise to torsion through nonminimal couplings and/or second-order derivatives of $\phi$. The EH four-form term is given by

$$
\mathcal{L}_{\mathrm{EH}}^{(4)}(e, \omega)=\frac{1}{4 \kappa_{4}} \epsilon_{a b c d} R^{a b} \wedge e^{c} \wedge e^{d},
$$

and therefore the field equations take the form

$$
\begin{aligned}
& \delta_{e} \mathcal{L}^{(4)}(e, \omega, \phi)=\delta_{e} \mathcal{L}_{\mathrm{EH}}^{(4)}+\delta_{e}(\text { other terms })=0 \\
& \delta_{\omega} \mathcal{L}^{(4)}(e, \omega, \phi)=\delta_{\omega} \mathcal{L}_{\mathrm{EH}}^{(4)}+\delta_{\omega}(\text { other terms })=0 \\
& \delta_{\phi} \mathcal{L}^{(4)}(e, \omega, \phi)=\delta_{\phi}(\text { other terms })=0
\end{aligned}
$$

where

$$
\begin{aligned}
\delta_{e} \mathcal{L}_{\mathrm{EH}}^{(4)}(e, \omega) & =\frac{1}{2 \kappa_{4}} \epsilon_{a b c d} R^{a b} \wedge e^{c} \wedge \delta e^{d} \\
\delta_{\omega} \mathcal{L}_{\mathrm{EH}}^{(4)}(e, \omega) & =\frac{1}{2 \kappa_{4}} \epsilon_{a b c d} \delta \omega^{a b} \wedge T^{c} \wedge e^{d}
\end{aligned}
$$

We consider now a background configuration $\bar{e}^{a}, \bar{\omega}^{a b}$, $\bar{\phi}$ satisfying the field equations (84)turbations around it as in eqs. (64)- When doing 
this, the result reads

$$
\begin{aligned}
\mathcal{G} & +\frac{1}{4 \kappa_{4}} \epsilon_{a b c d} \bar{R}^{a b} \wedge h^{c} \wedge \delta e^{d}+ \\
+\frac{1}{2 \kappa_{4}} \epsilon_{a b c d} \overline{\mathrm{D}} \mathcal{V}^{a b} & \wedge \bar{e}^{c} \wedge \delta e^{d}+
\end{aligned}
$$$$
+(\text { linear perturbations of other terms })=0 \text {, }
$$

where the four-form $\mathcal{G}$ is given by

$$
\mathcal{G}=\frac{1}{2 \kappa_{4}} \epsilon_{a b c d} \overline{\mathrm{D}} \mathcal{U}^{a b} \wedge \bar{e}^{c} \wedge \delta e^{d} .
$$

The $\mathcal{G}$-term generates a gravitational wave described by the generalized wave operator [cf. eq. (57)] $\mathbf{\square}_{\mathrm{dR}}=\mathrm{D}^{\ddagger} \mathrm{D}+$ $\mathrm{DD}^{\ddagger}$ coupled with torsion in a nontrivial way, in strong contrast with the example of eq. (52).

Using eq. (79) in eq. (90), we get

$$
\begin{aligned}
\mathcal{G} & =-\frac{1}{4 \kappa_{4}} \bar{*}\left\{\overline{\mathcal{D}}^{a} \overline{\mathcal{D}}_{a} h_{d}-\overline{\mathcal{D}}_{a} \overline{\mathcal{D}}_{d} h^{a}+\right. \\
& -\bar{e}^{c} \overline{\mathcal{D}}_{c}\left(\bar{\Sigma}_{d} \overline{\mathcal{D}}_{a} h^{a}-\overline{\mathcal{D}}_{d} h\right)+ \\
& -\frac{1}{2}\left[\bar{\Sigma}_{b}\left(\overline{\mathcal{D}}^{a} \overline{\mathcal{D}}_{a} h^{b}-\overline{\mathcal{D}}_{a} \overline{\mathcal{D}}^{b} h^{a}\right)+\right. \\
& \left.\left.-\overline{\mathcal{D}}_{b}\left(\bar{\Sigma}^{b} \overline{\mathcal{D}}_{a} h^{a}-\overline{\mathcal{D}}^{b} h\right)\right] \bar{e}_{d}\right\} \wedge \delta e^{d},
\end{aligned}
$$

where $\bar{*}$ stands for the Hodge dual under the background metric structure associated to $\bar{e}^{a}, \overline{\mathcal{D}}^{a}=\bar{\Sigma}^{a} \overline{\mathrm{D}}+\overline{\mathrm{D}} \bar{\Sigma}^{a}$, with the operator $\bar{\Sigma}^{a}=-\bar{*}\left(\bar{e}^{a} \wedge \bar{*}\right.$, and $h=\bar{\Sigma}_{a} h^{a}$ (see Table【 for a summary of the different derivatives defined in this paper).

Let $\zeta$ be a vector field. An infinitesimal Lie dragging $1-£_{\zeta}$ generated by $\zeta$ on the background geometry corresponds to

$h_{a b} \rightarrow h_{a b}^{\prime}=h_{a b}-\left(\overline{\mathcal{D}}_{a} \zeta_{b}+\overline{\mathcal{D}}_{b} \zeta_{a}\right)+\zeta^{c} \bar{\Sigma}_{c}\left(\bar{\Sigma}_{a} \bar{T}_{b}+\bar{\Sigma}_{b} \bar{T}_{a}\right)$.

Performing the standard change of variable $h^{a} \rightarrow \tilde{h}^{a}$,

$$
h^{a}=\tilde{h}^{a}-\frac{1}{2} \bar{e}^{a} \tilde{h},
$$

where $\tilde{h}$ is the trace of the new Lorentz vector oneform variable $\tilde{h}^{a}$, it is possible to prove that under (92), the "divergence" $\overline{\mathcal{D}}_{a} \tilde{h}^{a}=\left(\bar{\Sigma}_{a} \overline{\mathrm{D}}+\overline{\mathrm{D}} \bar{\Sigma}_{a}\right) \tilde{h}^{a}$ transforms as $\overline{\mathcal{D}}_{a} \tilde{h}^{a} \rightarrow \overline{\mathcal{D}}_{a} \tilde{h}^{\prime a}$, where $\overline{\mathcal{D}}_{a} \tilde{h}^{\prime a}$ is given by

$$
\begin{aligned}
\overline{\mathcal{D}}_{a} \tilde{h}^{a} & =\left[-\stackrel{\circ}{\mathcal{D}}_{a} \stackrel{\circ}{\mathcal{D}}^{a} \zeta_{b}+\overline{\mathcal{D}}_{a} \bar{\Sigma}_{b} \tilde{h}^{a}-\bar{\Sigma}_{a b} \stackrel{\circ}{\bar{R}}^{a}{ }_{c} \zeta^{c}+\right. \\
& -\bar{\Sigma}^{a c} \bar{T}_{a}\left(\stackrel{\circ}{\mathcal{D}}_{c} \zeta_{b}+\stackrel{\circ}{\mathcal{D}}_{b} \zeta_{c}-\eta_{c b} \stackrel{\circ}{\mathcal{D}}_{p} \zeta^{p}\right)+ \\
& \left.+\bar{\Sigma}_{c b} \bar{T}_{a} \bar{\Sigma}^{c} \tilde{h}^{a}\right] \bar{e}^{b}
\end{aligned}
$$

with $\stackrel{\circ}{\overline{\mathcal{D}}_{a}}=\bar{\Sigma}_{a} \stackrel{\circ}{\bar{D}}+\stackrel{\circ}{\bar{D}} \bar{\Sigma}_{a}$. This means we can always choose the "Lorenz gauge"

$$
\overline{\mathcal{D}}_{a} \tilde{h}^{a}=0
$$

with a vector field $\zeta$ such that the right-hand side of eq. (94) vanishes.
Choosing this gauge and using eqs. (62)-(63), it is possible to recast eq. (89) in terms of $\tilde{h}^{a}$ as

$$
\begin{gathered}
\overline{\mathbf{Q}}_{\mathrm{dR}} \tilde{h}^{d}+\bar{\Sigma}_{a d}\left(\bar{R}_{b}^{a} \wedge \tilde{h}^{b}\right)+ \\
-\left\{A_{d}+B_{d}+\frac{1}{2} \bar{e}_{d}\left[C-\bar{\Sigma}_{c}\left(A^{c}+B^{c}\right)\right]\right\}+ \\
+\epsilon_{a b c d} \bar{*}\left(\bar{R}^{a b} \wedge h^{c}+2 \overline{\mathrm{D}} \mathcal{V}^{a b} \wedge \bar{e}^{c}\right)+ \\
+(\text { linear perturbations of other terms })=0,
\end{gathered}
$$

where $\overline{\mathbf{D}}_{\mathrm{dR}} \tilde{h}_{d}$ is given by the generalized Weitzenböck identity (58),

$$
\overline{\mathbf{D}}_{\mathrm{dR}} \tilde{h}_{a}=-\overline{\mathcal{D}}^{b} \overline{\mathcal{D}}_{b} \tilde{h}_{a}+\bar{\Sigma}_{b}\left(\bar{R}^{b}{ }_{c} \bar{\Sigma}^{c} \tilde{h}_{a}-\bar{R}_{a}^{c} \bar{\Sigma}^{b} \tilde{h}_{c}\right),
$$

and $A_{a}, B_{a}$ and $C$ are the torsional terms

$$
\begin{aligned}
A_{a} & =\left(\bar{\Sigma}_{c a} \bar{T}_{b}\right) \overline{\mathcal{D}}^{b} \tilde{h}^{c}+\tilde{h}^{b c} \overline{\mathrm{D}} \bar{\Sigma}_{c a} \bar{T}_{b}, \\
B_{a} & =\left(\bar{\Sigma}_{c} \bar{T}_{b}\right) \bar{\Sigma}^{b}\left[\overline{\mathcal{D}}_{a}\left(\tilde{h} \bar{e}^{c}\right)-\overline{\mathcal{D}}^{c}\left(\tilde{h} \bar{e}_{a}\right)\right]+ \\
& +\frac{1}{2}\left\{\tilde{h} \overline{\mathrm{D}}^{\ddagger} \bar{T}_{a}+\bar{\Sigma}^{b}\left[\overline{\mathrm{D}}\left(\tilde{h} \bar{\Sigma}_{a} \bar{T}_{b}\right)-\bar{T}_{b} \bar{\Sigma}_{a} \overline{\mathrm{D}} \tilde{h}\right]\right\}, \\
C & =\overline{\mathcal{D}}^{c}\left(\tilde{h}^{a b} \bar{\Sigma}_{b c} \bar{T}_{a}\right)+\left(\bar{\Sigma}_{b c} \bar{T}_{a}\right) \bar{\Sigma}^{a} \overline{\mathcal{D}}^{c} \tilde{h}^{b} .
\end{aligned}
$$

The equation for the propagation of linear perturbations is found by replacing eq. (96) in eq. (89). Doing so, we observe that in the context of nonvanishing torsion:

- The metric wave $\tilde{h}_{a b}$ couples to both, the background torsion and the background curvature.

- The metric wave $\tilde{h}_{a b}$ couples to an independent propagating torsion wave mode, $\mathcal{V}^{a b}$.

- Some of the coupling between $\tilde{h}_{a b}$ and the background torsion occurs through the trace $\tilde{h}$. All this dependence has been "packed" in the Lorentzvector one-form $B_{a}$, but the important point is that the "traceless" variable $\tilde{h}_{a b}$ no longer leads to equations without the trace $\tilde{h}$.

\section{Gravitational Waves and generic terms of the Horndeski Lagrangian}

In section IVB we showed that the $\mathrm{EH}$ term in the Horndeski Lagrangian can produce gravitational waves interacting with the background torsion and propagating torsional modes. In this section we highlight those other terms in the Horndeski family that can lead to similar behavior.

Generic terms will couple $\tilde{h}^{a}, \mathcal{V}^{a b}$ and $\varphi$ with the background curvature $\bar{R}^{a b}$ and torsion $\bar{T}^{a}$, but only some very specific terms will contribute with second-order wavelike operators on the metric mode $\left(\partial^{2} \tilde{h}^{a}\right.$ terms), and first-order operators acting on the torsional mode $\left(\partial \mathcal{V}^{a b}\right.$ terms). 
For the sake of simplicity, let us focus on linear perturbations of the vierbein and spin connection, leaving the scalar field unchanged (i.e., $\varphi=0$ ). In this case, the linear perturbations on $e^{a}, R^{a b}, T^{a}, Z^{a}, \theta^{a}$ and $\pi^{a}$ (the fundamental ingredients of the field equations) read

$$
\begin{aligned}
\bar{e}^{a} \rightarrow e^{a} & =\bar{e}^{a}+\frac{1}{2} h^{a}, \\
\bar{R}^{a b} \rightarrow R^{a b} & =\bar{R}^{a b}+\overline{\mathrm{D}} \mathcal{V}^{a b}-\frac{1}{2} \overline{\mathrm{D}}\left(\bar{\Sigma}^{a} \overline{\mathrm{D}} h^{b}-\bar{\Sigma}^{b} \overline{\mathrm{D}} h^{a}\right) \\
\bar{T}^{a} \rightarrow T^{a} & =\bar{T}^{a}+\mathcal{V}^{a}{ }_{b} \wedge \bar{e}^{b}-\frac{1}{2} \bar{\Sigma}^{a}\left(h_{b} \wedge \bar{T}^{b}\right) \\
\bar{Z}^{a} \rightarrow Z^{a} & =\bar{Z}^{a}-\frac{1}{2} h^{a}{ }_{b} \bar{Z}^{b} \\
\bar{\theta}^{a} \rightarrow \theta^{a} & =\bar{\theta}^{a}-\frac{1}{2} h^{a}{ }_{b} \bar{\theta}^{b}, \\
\bar{\pi}^{a} \rightarrow \pi^{a} & =\bar{\pi}^{a}-\frac{1}{2} h^{a}{ }_{b} \bar{\pi}^{b}+ \\
& +\left[\mathcal{V}^{a b}-\frac{1}{2}\left(\bar{\Sigma}^{a} \overline{\mathrm{D}} h^{b}-\bar{\Sigma}^{b} \overline{\mathrm{D}} h^{a}-\overline{\mathrm{D}} h^{a b}\right)\right] \bar{Z}_{b} .
\end{aligned}
$$

In the above equations, only the perturbation of the Lorentz curvature includes second-order derivatives of $h^{a}$ (through the operator $\overline{\mathrm{D}} \bar{\Sigma}^{a} \overline{\mathrm{D}}$ ) and first-order derivatives of the torsional perturbation $\overline{\mathrm{D}} \mathcal{V}^{a b}$. Given that $\mathrm{d}^{2}=0$, and that the Hodge operator appears in the Lagrangian only through $\Sigma^{a}=-* e^{a} \wedge(*$, we find that in the equations of motion $\mathcal{E}_{a b}=0$ and $\mathcal{E}_{a}=0$ [cf. eqs. (25) and (26)] $\partial^{2} \tilde{h}^{a}$ and $\partial \mathcal{V}^{a b}$ can arise only from terms where the curvature is present.

In the Horndeski Lagrangian, this boils down to (i) terms where the Lorentz curvature appears explicitly,

$$
\begin{aligned}
& (F+2 W) \epsilon_{a b c d} R^{a b} \wedge e^{c} \wedge e^{d} \\
& \kappa_{3} \epsilon_{a b c d} R^{a b} \wedge e^{c} \wedge \theta^{d} \\
& \kappa_{1} \epsilon_{a b c d} R^{a b} \wedge e^{c} \wedge \pi^{d}
\end{aligned}
$$

and (ii) any terms having two or more $\pi^{a}$ 's:

$$
\begin{aligned}
& \frac{\partial \kappa_{1}}{\partial X} \epsilon_{a b c d} \pi^{a} \wedge \pi^{b} \wedge \pi^{c} \wedge e^{d}, \\
& \frac{\partial \kappa_{3}}{\partial X} \epsilon_{a b c d} \theta^{a} \wedge \pi^{b} \wedge \pi^{c} \wedge e^{d}, \\
& \frac{\partial F}{\partial X} \epsilon_{a b c d} \pi^{a} \wedge \pi^{b} \wedge e^{c} \wedge e^{d} .
\end{aligned}
$$

These three last terms produce curvature in the field equations through the Bianchi identity, $\mathrm{D} \pi^{a}=\mathrm{D}^{2} Z^{a}=$ $R^{a}{ }_{b} Z^{b}$. This can most easily be seen by considering the dependence of $Z^{a}=\Sigma^{a} \mathrm{~d} \phi$ on the vierbein and integrating by parts [see, e.g., eq. (31)].

As an example, let us consider the term

$$
\mathcal{L}_{\theta}=\frac{1}{2} \epsilon_{a b c d} R^{a b} \wedge e^{c} \wedge \theta^{d} .
$$

The variation of $\mathcal{L}_{\theta}$ under an infinitesimal change in the vierbein reads

$$
\delta_{e} \mathcal{L}_{\theta}=\left[\frac{1}{2} \epsilon_{a b c d} R^{a b} \wedge \theta^{c}+\Sigma^{a}\left(\mathcal{G}_{a} \wedge \theta_{d}\right)\right] \wedge \delta e^{d},
$$

where the "Einstein tensor" three-form $\mathcal{G}_{d}$ is given by

$$
\mathcal{G}_{d}=\frac{1}{2} \epsilon_{a b c d} R^{a b} \wedge e^{c}
$$

Under linear perturbations, $\delta_{e} \mathcal{L}_{\theta}$ behaves as

$$
\begin{aligned}
\delta_{e} \mathcal{L}_{\theta} & =\delta_{\bar{e}} \overline{\mathcal{L}}_{\theta}+\left\{\bar{\Sigma}^{m}\left(\mathcal{W}_{m} \wedge \bar{\theta}_{d}+\overline{\mathcal{G}}_{m} \wedge \Upsilon_{d}\right)+\right. \\
& +\frac{1}{2} \epsilon_{a b c d} \bar{R}^{a b} \wedge \Upsilon^{c}+ \\
& +\frac{1}{2} \epsilon_{a b c d} \overline{\mathrm{D}}\left[\mathcal{V}^{a b}-\frac{1}{2}\left(\bar{\Sigma}^{a} \overline{\mathrm{D}} h^{b}-\bar{\Sigma}^{b} \overline{\mathrm{D}} h^{a}\right)\right] \wedge \bar{\theta}^{c}+ \\
& \left.-\frac{1}{2} h^{m n} \bar{\Sigma}_{n}\left(\overline{\mathcal{G}}_{m} \wedge \bar{\theta}_{d}\right)\right\} \wedge \delta e^{d},
\end{aligned}
$$

where $\Upsilon^{a}=-\frac{1}{2} h^{a}{ }_{b} \bar{\theta}^{b}+\mathrm{d} \bar{\phi} \bar{\Sigma}^{a} \mathrm{~d} \varphi+\bar{Z}^{a} \mathrm{~d} \varphi$ is the linear perturbation of $\theta^{a}$ and $\mathcal{W}_{a}$ is just a shortcut for the gravitational wave terms we have already seen in eq. (96),

$$
\begin{aligned}
\mathcal{W}_{d} & =-\frac{1}{4} \epsilon_{a b c d} \overline{\mathrm{D}}\left(\bar{\Sigma}^{a} \overline{\mathrm{D}} h^{b}-\bar{\Sigma}^{b} \overline{\mathrm{D}} h^{a}\right) \wedge \bar{e}^{c}+ \\
& +\frac{1}{2} \epsilon_{a b c d}\left(\frac{1}{2} \bar{R}^{a b} \wedge h^{c}+\overline{\mathrm{D}} \mathcal{V}^{a b} \wedge \bar{e}^{c}\right) \\
& =\frac{1}{4} \bar{*}\left[\overline{\boldsymbol{a}}_{\mathrm{dR}} \tilde{h}_{d}+\bar{\Sigma}_{a d}\left(\bar{R}^{a}{ }_{b} \wedge \tilde{h}^{b}\right)\right]+ \\
& -\frac{1}{4} \bar{*}\left\{A_{d}+B_{d}+\frac{1}{2} \bar{e}_{d}\left[C-\bar{\Sigma}_{c}\left(A^{c}+B^{c}\right)\right]\right\}+ \\
& +\frac{1}{2} \epsilon_{a b c d}\left(\frac{1}{2} \bar{R}^{a b} \wedge h^{c}+\overline{\mathrm{D}} \mathcal{V}^{a b} \wedge \bar{e}^{c}\right),
\end{aligned}
$$

where $A_{a}, B_{a}$ and $C$ are the torsion couplings defined in eqs. (98)- (100).

Similar expressions for the linear perturbations of terms like (109)-113) can also be found.

Beyond the complicated algebra, the interesting point is that, quite generally, every appearance of $\partial^{2} \tilde{h}^{a}$ terms is related with couplings with torsion. In the context of the Horndeski Lagrangian, the coupling between $\partial^{2} \tilde{h}^{a}$ terms and torsion seems to be rather the rule than the exception.

\section{CONCLUSIONS}

When YM bosons are described by connections on fiber bundles, their field strength is given by $F=\mathrm{d} A+\frac{1}{2}[A, A]$, regardless of the curvature and torsion of the spacetime (basis) manifold. The YM Lagrangian, $\mathcal{L}_{\mathrm{YM}}=$ $-\frac{1}{4}\langle F \wedge * F\rangle$, only has information about the connection $A$ and the background spacetime metric $g_{\mu \nu}$ needed to construct the Hodge $*$-operator. Therefore, YM bosons 
will be sensitive to the spacetime Riemann (metric) curvature but oblivious to torsion. Of all Standard Model fields, torsion only interacts, albeit very weakly, with fermions in the ECSK theory. Since it is always possible to "pack" torsional terms in an effective stress-energy tensor, it may seem tempting to consider torsion as a dark matter candidate (see, e.g., Ref. [48]).

Adopting geometry as a solution to the dark matter problem is an idea with a rich history (see, e.g., Ref. 49]). There are, however, at least two potential weak points worth considering:

- In the pure ECSK theory, torsion does not propagate in vacuum and fermions are its only (very weak) source. Therefore, in order to consider the idea seriously it is necessary to look for more general theories in $d=4$ and new torsion sources.

- The same "darkness" of torsion (i.e., its lack of interaction with YM fields) that makes the idea attractive also makes it hard to falsify in any foreseeable accelerator physics experiment. Therefore it seems appropriate to find a torsion-sensitive phenomenon outside of the Standard Model in order to test the idea of torsion as dark matter.

In this paper we have explored solutions to both of these issues. Regarding the first point, in section II we take Horndeski's theory and allow it to develop nonzero torsion by recasting it in Cartan's first-order formalism. The main result of this exercise is that every nonminimal coupling of the geometry with $\phi$ and every term in the Lagrangian with second derivatives of $\phi$ are generic sources of torsion. This was to be expected in the light of previous work, such as section 1.7.1 of Ref. [45] on the Brans-Dicke theory and Ref. [21] on nonminimal coupling with the Gauss-Bonnett term. The main novelty of section II is the development of new mathematical techniques based on the properties of the $\Sigma^{a}$ operator, making it accessible to work with the full Horndeski Lagrangian in first-order formalism and without imposing the torsionless condition.

In section [V] we explored the idea of using gravitational waves as a probe for torsion, and in section III we introduced the necessary mathematical tools to address this problem. In particular, in section III we developed a generalization of the Laplace-de Rham operator, $\square_{\mathrm{dR}}=\mathrm{d}^{\dagger} \mathrm{d}+\mathrm{dd}^{\dagger}$, to a new operator $\boldsymbol{\square}_{\mathrm{dR}}=\mathrm{D}^{\ddagger} \mathrm{D}+\mathrm{DD}^{\ddagger}$ which acts covariantly on $p$-forms with Lorentz indices, where $\mathrm{D}^{\ddagger}=-\Sigma_{a} \mathrm{D} \Sigma^{a}$. In section IV we showed that any Horndeski Lagrangian that includes the EH term will give rise to gravitational waves, governed by the $\mathbf{a}_{\mathrm{dR}}$ operator, plus new interactions with the background torsion.

The following is an incomplete list of the many problems that remain open for future work.

- It is clear that the Horndeski theorem breaks down in the case of nonvanishing torsion: there are many new torsional terms which can be added to the Lagrangian which give rise only to second-order field equations. What is the most general Lagrangian for this case remains as an open problem.

- We have shown in section [V] that gravitational waves interact with the background torsion, and a new torsional mode appears. However, the phenomenology of this interaction still remains to be modeled. Even further, in any realistic astrophysical scenario it is necessary to go up to second-order in perturbations (see, e.g., Ref. [50]).

- It is not yet clear which, if any, of the Horndeski family members generate suitable dark matter profiles. With sufficiently precise observations, one may hope to use this information to select the most appropriate Lagrangians, or at least rule some of them out. The same is true regarding gravitational waves propagation. Some ideas have been proposed about this point in Ref. [51], but only for the torsionless case.

- The cosmological implications of Horndeski's theory have been studied only on particular cases (see, e.g., Refs. [15, 17, 18, 21]).

\section{ACKNOWLEDGMENTS}

We are grateful to Antonella Cid, José M. Izquierdo, Patricio Mella, Julio Oliva, Patricio Salgado, and Jorge Zanelli for many enlightening conversations. This research was partially funded by Fondecyt grants 1130653 , 1150719 (FI), and 3160437 (OV), and by Conicyt scholarships 72160340 (FC-T), 21160784 (JB), 21161574 (PM), and 21161099 (DN) from the Government of Chile. ER wishes to thank the German Academic Exchange Service (DAAD) for financial support, and Dieter Lüst for his kind hospitality at the Arnold Sommerfeld Center for Theoretical Physics in Munich.
[1] Gregory Walter Horndeski, "Second-order scalartensor field equations in a four-dimensional space," Int. J. Theor. Phys. 10, 363-384 (1974).

[2] Christos Charmousis, Edmund J. Copeland, Antonio Padilla, and Paul M. Saffin, "General second order scalar-tensor theory, self tuning, and the Fab Four," Phys. Rev. Lett. 108, 051101 (2012) arXiv:1106.2000 [hep-th]

[3] Alberto Nicolis, Riccardo Rattazzi, and Enrico Trincherini, "The Galileon as a local modification of gravity," Phys. Rev. D 79, 064036 (2009) arXiv:0811.2197 [hep-th] 
[4] Tsutomu Kobayashi, Masahide Yamaguchi, and Jun'ichi Yokoyama, "Generalized G-inflation: Inflation with the most general second-order field equations," Prog. Theor. Phys. 126, 511-529 (2011). arXiv:1105.5723 [hep-th].

[5] Sourav Bhattacharya and Sumanta Chakraborty, "Constraining some Horndeski gravity theories," $\quad$ Phys. Rev. D 95, 044037 (2017) arXiv:1607.03693 [gr-qc]

[6] Nikodem J. Popławski, "Cosmology with torsion: An alternative to cosmic inflation," Phys. Lett. B 694, 181-185 (2010), [Erratum: Phys. Lett. B 701, 672 (2011)], arXiv:1007.0587 [astro-ph.CO]

[7] Friedrich W. Hehl, Paul von der Heyde, G. David Kerlick, and James M. Nester, "General relativity with spin and torsion: Foundations and prospects," Rev. Mod. Phys. 48, 393-416 (1976).

[8] Daniel Z. Freedman and Antoine Van Proeyen, Supergravity, 1st ed. (Cambridge University Press, 2012).

[9] G. David Kerlick, "Cosmology and particle pair production via gravitational spin-spin interaction in the Einstein-Cartan-Sciama-Kibble theory of gravity," Phys. Rev. D 12, 3004-3006 (1975).

[10] Dirk Puetzfeld, "Status of non-Riemannian cosmology," Proceedings, 6th UCLA Symposium on Sources and Detection of Dark Matter and Dark Energy in the Universe: Marina del Rey, CA, USA, February 18-20, 2004, New Astron. Rev. 49, 59-64 (2005), arXiv:gr-qc/0404119 [gr-qc]

[11] E. Sezgin and P. van Nieuwenhuizen, "New ghostfree gravity Lagrangians with propagating torsion," Phys. Rev. D 21, 3269-3280 (1980).

[12] E. Sezgin, "Class of ghost-free gravity Lagrangians with massive or massless propagating torsion," Phys. Rev. D 24, 1677-1680 (1981).

[13] Lay Nam Chang, Oleg Lebedev, Will Loinaz, and Tatsu Takeuchi, "Universal torsion induced interaction from large extra dimensions," Phys. Rev. Lett. 85, 3765-3768 (2000), arXiv:hep-ph/0005236 [hep-ph]

[14] Oleg Lebedev, "Torsion constraints in the RandallSundrum scenario," Phys. Rev. D 65, 124008 (2002), arXiv:hep-ph/0201125 [hep-ph]

[15] R. Jackiw and S. Y. Pi, "Chern-Simons modification of general relativity," Phys. Rev. D 68, 104012 (2003), arXiv:gr-qc/0308071 [gr-qc]

[16] Yuri N. Obukhov, "Poincare gauge gravity: Selected topics," Int. J. Geom. Meth. Mod. Phys. 3, 95-138 (2006), arXiv:gr-qc/0601090 [gr-qc]

[17] Marcelo Botta Cantcheff, "Einstein-Cartan formulation of Chern-Simons Lorentz Violating Gravity and Holographic Structure," Phys. Rev. D 78, 025002 (2008). arXiv:0801.0067 [hep-th].

[18] Umit Ertem and Özgür Açı, "Generalized Chern-Simons Modified Gravity in First-Order Formalism," Gen. Rel. Grav. 45, 477-488 (2013), arXiv:0912.1433 [gr-qc].

[19] Peter Baekler and Friedrich W. Hehl, "Beyond EinsteinCartan gravity: Quadratic torsion and curvature invariants with even and odd parity including all boundary terms," Class. Quant. Grav. 28, 215017 (2011), arXiv:1105.3504 [gr-qc]

[20] Milutin Blagojević and Friedrich W. Hehl, eds.,
Gauge Theories of Gravitation (Imperial College Press, London, 2013).

[21] Adolfo Toloza and Jorge Zanelli, "Cosmology with scalar-Euler form coupling," Class. Quant. Grav. 30, 135003 (2013) arXiv:1301.0821 [gr-qc].

[22] Oscar Castillo-Felisola, Cristobal Corral, Cristian Villavicencio, and Alfonso R. Zerwekh, "Fermion Masses Through Condensation in Spacetimes with Torsion," Phys. Rev. D 88, 124022 (2013) arXiv:1310.4124 [hep-ph].

[23] Oscar Castillo-Felisola, Cristobal Corral, Ivan Schmidt, and Alfonso R. Zerwekh, "Updated limits on extra dimensions through torsion and LHC data," Mod. Phys. Lett. A29, 1450081 (2014) arXiv:1404.5195 [hep-ph]

[24] Oscar Castillo-Felisola, Cristobal Corral, Sergey Kovalenko, and Ivan Schmidt, "Torsion in Extra Dimensions and One-Loop Observables," $\quad$ Phys. Rev. D 90, 024005 (2014) arXiv:1405.0397 [hep-ph]

[25] R. T. Hammond, "Torsion gravity," Rept. Prog. Phys. 65, 599-649 (2002)

[26] I. L. Shapiro, "Physical aspects of the spacetime torsion," Phys. Rept. 357, 113 (2002), arXiv:hep-th/0103093 [hep-th].

[27] I. M. Benn, T. Dereli, and R. W. Tucker, "Gauge Field Interactions in Spaces With Arbitrary Torsion," Phys. Lett. B96, 100-104 (1980).

[28] M. Novello, "Scalar and Massless Vector Fields in Cartan Space," Phys. Lett. A59, 105-106 (1976).

[29] S. Hojman, M. Rosenbaum, M. P. Ryan, and L. C. Shepley, "Gauge invariance, minimal coupling, and torsion," Phys. Rev. D 17, 3141-3146 (1978)

[30] V. De Sabbata and M. Gasperini, "On The Maxwell Equations In A Riemann-Cartan Space," Phys. Lett. A77, 300-302 (1980)

[31] A. H. Chamseddine, "Topological gravity and supergravity in various dimensions," Nucl. Phys. B 346, 213-234 (1990).

[32] Patricio Salgado, Patricio Salgado-Rebolledo, and Omar Valdivia, "Topological gravity and gauged Wess-Zumino-Witten term," $\quad$ Phys. Lett. B 728, 99-104 (2014), arXiv:1311.2532 [math-ph].

[33] Patricio Salgado, Richard J. Szabo, and Omar Valdivia, "Topological gravity and transgression holography," Phys. Rev. D 89, 084077 (2014), arXiv:1401.3653 [hep-th]

[34] S. Salgado, F. Izaurieta, N. González, and G. Rubio, "Gauged Wess-Zumino-Witten actions for generalized Poincaré algebras," Phys. Lett. B 732, 255-262 (2014).

[35] P. Catalán, F. Izaurieta, P. Salgado, and S. Salgado, "Topological gravity and Chern-Simons forms in $d=4$," Phys. Lett. B 751, 205-208 (2015)

[36] Sourav Sur and Arshdeep Singh Bhatia, "Weakly dynamic dark energy via metric-scalar couplings with torsion," (2016), arXiv:1611.00654 [gr-qc].

[37] Ahmet Baykal and Tekin Dereli, "Linearized gravity in terms of differential forms," $\quad$ Eur. Phys. J. Plus 132, 52 (2017), arXiv:1612.05510 [gr-qc]

[38] Jérôme Gleyzes, David Langlois, Federico Piazza, and Filippo Vernizzi, "Healthy theories beyond 
Horndeski," Phys. Rev. Lett. 114, 211101 (2015) arXiv:1404.6495 [hep-th].

[39] David Langlois and Karim Noui, "Degenerate higher derivative theories beyond Horndeski: evading the Ostrogradski instability," JCAP 1602, 034 (2016) arXiv:1510.06930 [gr-qc]

[40] Jibril Ben Achour, Marco Crisostomi, Kazuya Koyama, David Langlois, Karim Noui, and Gianmassimo Tasinato, "Degenerate higher order scalar-tensor theories beyond Horndeski up to cubic order," JHEP 12, 100 (2016), arXiv:1608.08135 [hep-th]

[41] Jose María Ezquiaga, Juan García-Bellido, and Miguel Zumalacárregui, "Towards the most general scalar-tensor theories of gravity: a unified approach in the language of differential forms," Phys. Rev. D 94, 024005 (2016) arXiv:1603.01269 [hep-th]

[42] D. Lovelock, "The Einstein tensor and its generalizations," J. Math. Phys. 12, 498 (1971)

[43] Bruno Zumino, "Gravity theories in more than four dimensions," Phys. Rep. 137, 109-114 (1986)

[44] Alberto Iglesias, Nemanja Kaloper, Antonio Padilla, and Minjoon Park, "How (Not) to Palatini," Phys. Rev. D 76, 104001 (2007), arXiv:0708.1163 [astro-ph]

[45] Leonardo Castellani, Riccardo D'Auria, and Pietro Fré, eds. Supergravity and Superstrings: A Geometric Perspective Vol. 1: Mathematical Foundations (World Scientific,
1991).

[46] Pedro D. Alvarez, Pablo Pais, Eduardo Rodríguez, Patricio Salgado-Rebolledo, and Jorge Zanelli, "Supersymmetric 3D model for gravity with $S U(2)$ gauge symmetry, mass generation and effective cosmological constant," Class. Quant. Grav. 32, 175014 (2015) arXiv:1505.03834 [hep-th]

[47] Fabrizio Cordonier-Tello, Fernando Izaurieta, Patricio Mella, and Eduardo Rodríguez, "RandallSundrum brane Universe as a ground state for ChernSimons gravity," Class. Quant. Grav. 33, 235004 (2016), arXiv:1506.05128 [hep-th]

[48] Alexander S. Belyaev, Marc C. Thomas, and Ilya L. Shapiro, "Torsion as a Dark Matter Candidate from the Higgs Portal," Phys. Rev. D 95, 095033 (2017) arXiv:1611.03651 [hep-ph]

[49] Erik P. Verlinde, "Emergent Gravity and the Dark Universe," SciPost Phys. 2, 016 (2017) arXiv:1611.02269 [hep-th]

[50] Michele Maggiore, Gravitational Waves, Vol. 1: Theory and Experiments (Oxford University Press, 2007).

[51] Dario Bettoni, Jose María Ezquiaga, Kurt Hinterbichler, and Miguel Zumalacárregui, "Speed of Gravitational Waves and the Fate of ScalarTensor Gravity," Phys. Rev. D 95, 084029 (2017) arXiv:1608.01982 [gr-qc] 